\title{
DESIGN AND EXPERIENCE WITH THE WS/HS ASSEMBLY MOVEMENT USING LABVIEW VIS, NATIONAL INSTRUMENT MOTION CONTROLLERS, AND COMPUMOTOR ELECTRONIC DRIVE UNITS AND MOTORS *
}

\author{
D. Barr, L. Day, J.D. Gilpatrick, M. Gruchalla, D. Martinez, J. O’Hara, R. Shurter, M. Stettler, R. \\ Valdiviez, Los Alamos National Laboratory
}

\begin{abstract}
The Low-Energy Demonstration Accelerator (LEDA), designed and built at the Los Alamos National Laboratory, is part of the Accelerator Production of Tritium (APT) program and provides a platform for measuring high-power proton beam-halo formation. The technique used for measuring the beam halo employs nine combination Wire Scanner and Halo Scraper (WS/HS) devices. This paper will focus on the experience gained in the use of National Instrument (NI) LabVIEW VIs and motion controllers, and Compumotor electronic drive units and motors. The base configuration couples a Compumotor motor driven by a Parker-Hannifin Gemini GT Drive unit. The drive unit is controlled by a NI PXI7344 controller card, which in turn is controlled by a PC running custom built NI LabVIEW VIs. The function of the control VI's is to interpret instructions from the main control system, the Experimental Physics and Industrial Control System (EPICS), and carry out the corresponding motion commands. The main control VI has to run all nineteen WS/HS motor axes used in the accelerator. A basic discussion of the main accelerator control system, EPICs which is hosted on a VXI platform, and its interface with the PC based LabVIEW motion control software will be included.
\end{abstract}

\section{BEAM CONFIGURATION}

A 52-quadrupole-magnet lattice at LEDA is used to study the formation of high-power proton-beam halo formation. Essential to this study are a series of wire scanner and halo scraper devices (collectively known as WS/HS). These devices are designed to measure the beam's transverse distribution. By generating intentional lattice mismatches, it is possible to view halo formation at downstream WS/HS $[1,2]$. The first WS/HS is located just after the fourth quadrupole magnet in the halo lattice and benchmarks the beam produced by the RFQ. This benchmark is used as a nominal input to the halo lattice. A set of four WS/HSs are located approximately halfway through the lattice. These provide phase-space information after the beam has debunched. The final set of four WS/HSs are located near the end of the halo lattice and are used to measure beam halo there. These are used to measure beam halo at a location very sensitive to halo formation.

The wire scanner portion of the WS/HS is used to produce a cross-sectional or transverse distribution of the

\footnotetext{
* Supported by US DOE, Office of Defense Programs, and the Office of Nuclear Energy, Science and Technology
}

beam core. The halo scraper consists of two graphitescraping devices (one for each side of the distribution)[3] to acquire the tails of the beam distribution. Both the wire and scraper for a given transverse axis, either horizontal or vertical, are mounted on a single assembly and thus positioned by a single motion control unit (MCU). The present paper will only concern itself with controlling a single MCU.

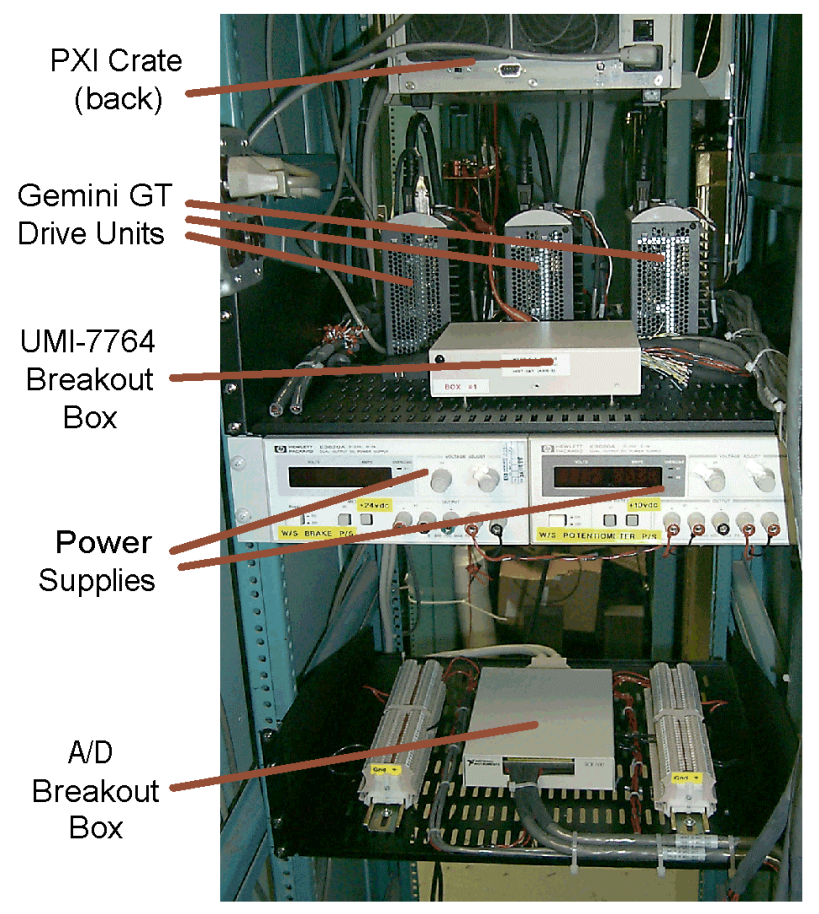

Figure 1: Electronics Setup for Three Axes

Each MCU comprises the mechanism needed to move one axis, either horizontal or vertical, at one longitudinal location in the halo lattice. A unit is comprised of a Compumotor motor driven by a Parker-Hannifin Gemini GT Drive unit. See Figure 1 for the basic layout for three of the nineteen axes. The drive unit is controlled by one channel of a four-channel National Instruments (NI) PXI7344 motion control card sitting in a NI PXI chassis. The PXI chassis is mapped to a PC via a NI MXI-3 interface mounted in a PCI slot in the PC. The PC runs a NI LabVIEW Virtual Instrument (VI). A VI is similar to a computer program, but runs in NI's LabVIEW graphical programming environment. The PC is in charge of all motion control for all WS/HS axes. There are a total of ten wire scanners while only nine WS/HSs. The 


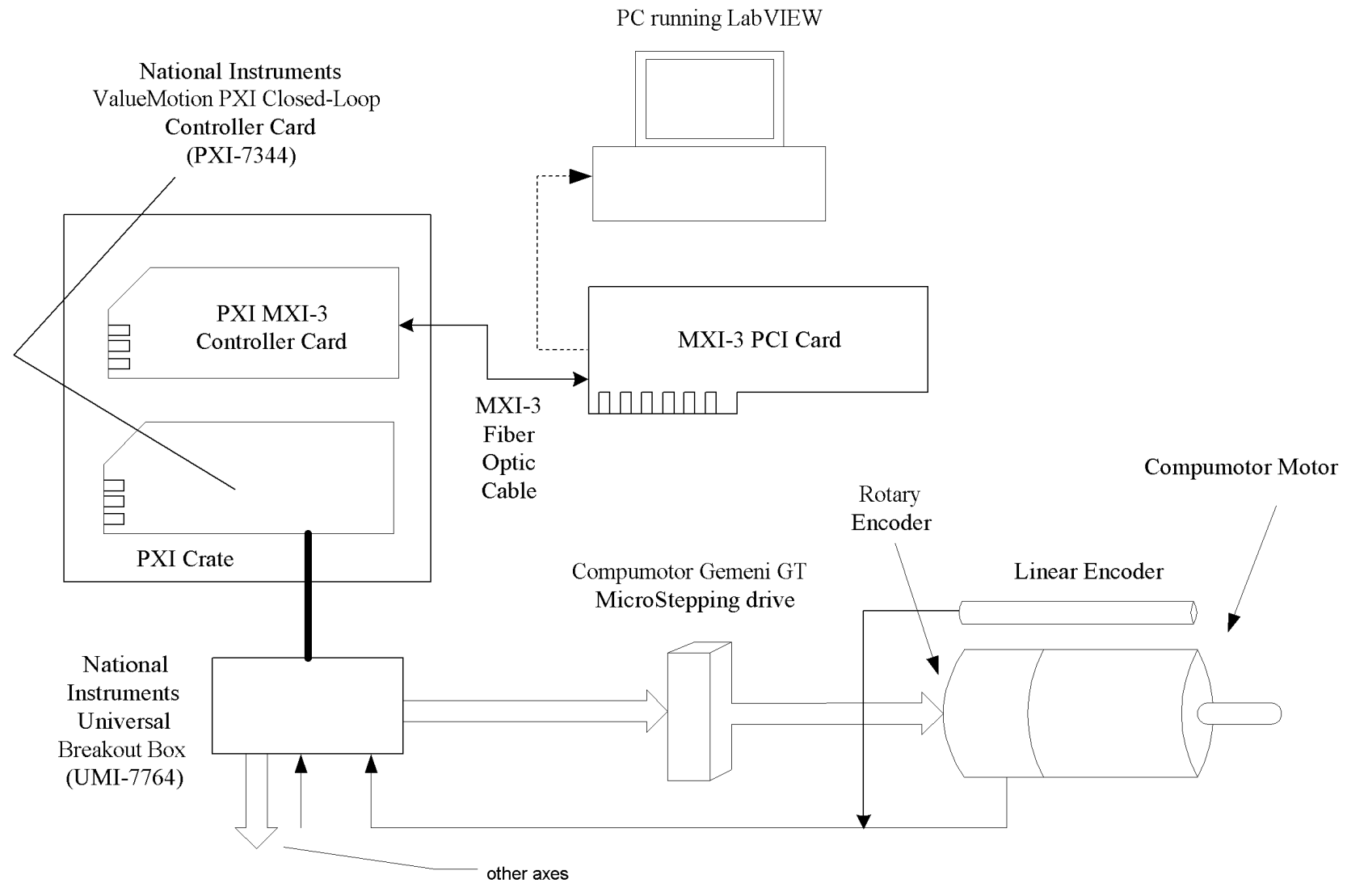

Figure 2: Basic Layout of Motion Control Unit with PC

missing WS/HS only uses one axis and hence one MCU. Five NI PXI-7344 cards are required to drive the resulting nineteen MCUs. One PC can easily handle control of these systems as only one axis is moved at a time. The motion control PC is controlled via Ethernet by the EPICS control system. Control is accomplished through the use of an ActiveX control schema. The basic system layout for one MCU and the PC is shown in Figure 2.

\section{MOTION CONTROL}

The NI PXI-7344 is a commercially available motion controller. In this application it is used as a four-axis closed-loop stepper motor controller running a simple PID control loop. The PXI-7344 is connected to a NI UMI7764 screw-terminal breakout box for connection with the drive unit and other feedback devices. An incremental linear optical encoder wired directly to the UMI-7764 achieves control feedback. An incremental rotary encoder attached directly to each motor is also included as a potential backup for a linear encoder failure, but as of this writing none has been needed. As the encoder used for feedback is incremental and hence not an absolute device, it will lose its position if power is ever dropped. For this reason, a linear potentiometer is also included. Since the value of resistance of the pot is fixed at a given spatial position, it operates as an absolute or non-volatile device. The pot value is entered into the system using a NI PXI$6031 \mathrm{E}$ A/D IO card. This interfaces to the pot wiper via a screw-terminal breakout box as in Figure 1. Although this is not used in the control loop, it does provide feedback to the operators in case of any problems, and acts as an independent analog input to the main accelerator run permit system. One problem encountered in system integration was that of motor holding noise [4]. Excessive motor holding noise corrupted the toroid signals, the wire scanner and scraper charge inputs, and the radiation beam loss systems. In order to compensate for this undesirable condition, it is necessary to disable the motor drive unit between successive moves for each scan. This is accomplished by a NI PXI-6527 Digital I/O card wired to the Gemini drive unit. Unfortunately, the drive units took more than 300 milliseconds to enable, thus removing the ability to take data at the desired $10 \mathrm{~Hz}$ beam repetition rate. This was to be done, by moving the scanner between consecutive beam pulses. As it turns out, this was not a hard requirement and the enable delay only extended the time for each scan. The typical time for a wire scan went from just under one minute to just under three minutes. 
The mechanical system was designed to safely counterbalance the assembly if a motor was to free run. In order to add extra safety, a brake was added and engaged whenever motor holding current is removed. A pair of limit switches is used for each axis. These are used for a startup position finding routine and as a motion safety control.

\section{EPICS INTERFACE}

The motion control PC is connected to the main accelerator control system (EPICS) by way of a TCP/IP ActiveX control written in LabVIEW. This control scheme interfaces with the EPICs Channel Access system [5]. A pair of EPICS process variable arrays are used to communicate between the EPICS control sequence and the motion control PC.

\section{TYPICAL RESULTS}

Figure 3 shows a basic test used to verify motion control unit operation. Depicted is commanded movement versus actual movement for 10 and 60 micron steps of a typical lattice scanner. The error is the difference between the commanded position and the actual measured position for a series of representative moves. The actual position was measured using the linear encoder. The MCU was moving at the nominal rate of $508 \mathrm{~mm}$ per second.

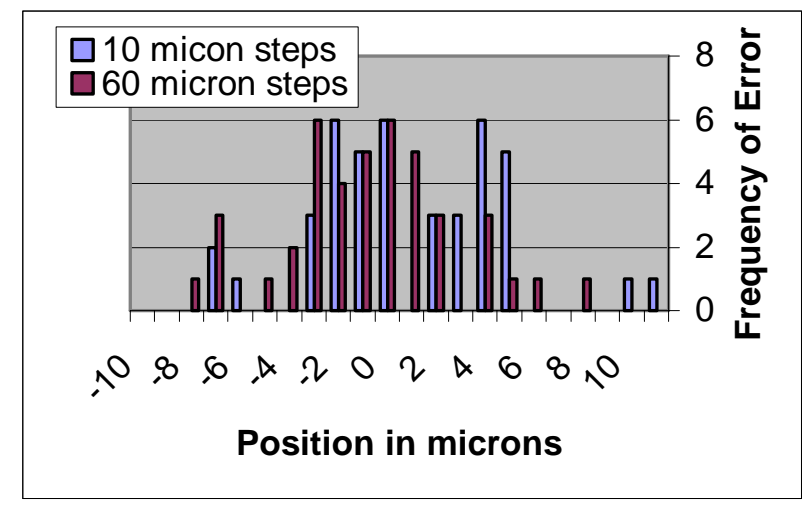

Figure 3: Histogram of Movement Errors for 10 and 60 micron step sizes

Looking at the raw data taken for Figure 3, it is determined that the rms value of the 10 micron step data is 4.08 microns and 3.66 microns for the 60 micron step data. This is less than $0.5 \%$ of a typical matched-beam rms width of $1 \mathrm{~mm}$ in both cases. From Figure 3, it is important to note that, although the distributions are similar, the position errors shown are in actual microns of position error and note a percentage of the requested step size. This indicates that the errors in stepping are fairly constant and negligibly dependent on step size. Thus at larger step sizes the percent error in each step decreases. Note that the typical step size for any given scrape in actual beam operations is 80 microns with a minimum of 70 microns. For wire scans the sizes ranged between 150 and 250 microns.

\section{SUMMARY}

The system worked extremely well and very reliably. There have been very few breakdowns and excellent performance of the EPICS Channel Access system. Overall maintenance has been minimal. The ability to work with a PC enables an easy to use, well understood, and low-cost alternative to more expensive and complex computing platforms.

\section{REFERENCES}

[1] J. D. Gilpatrick, et al., "Experience with the Low Energy Demonstration Accelerator (LEDA) Halo Experiment Beam Instrumentation," published at this conference.

[2] J. D. Gilpatrick, et al., " Beam-Profile Instrumentation for Beam-Halo Measurement: Overall Description and Operation," published at this conference.

[3] R. Valdiviez, et al., "The Final Mechanical Design, Fabrication, and Commissioning of a Wire Scanner and Scraper Assembly for Halo-Formation Measurements in a Proton Beam," published at this conference.

[4] M. Gruchalla, et al., "Beam Profile WireScanner/Halo-Scraper Sensor Analog Interface Electronics," published at the conference.

[5] L. Day, et al., "Automated Control and Real-Time Data Processing of Wire Scanner/Halo Scraper Measurements," published at this conference.

[6] J. D. Gilpatrick, et al., "Beam-Profile Instrumentation For A Beam-Halo Measurement: Overall Description, Operation, And Beam Data," DIPAC2001, Grenoble, France, May 13-15, 2001. 